\title{
Palm Fruit Juice Mitigates AZT Mitochondrial Genotoxicity and Dose- Dependent Cytotoxicity
}

\author{
Osborne $\mathrm{AE}^{1}$, Sanchez $\mathrm{JA}^{1^{*}}$, Solomon $\mathbf{M}^{2}$, Stopa $\mathbf{A}^{1}$, Wangh $\mathrm{LJ}^{1}$, Sambanthamurthi $\mathbf{R}^{3}$ and Hayes $\mathrm{KC}^{1}$ \\ ${ }^{1}$ Department of Biology, Brandeis University, Waltham, MA, 02454, USA \\ ${ }^{2}$ Molecular Pharmacology and Chemistry, Sloan-Kettering Institute, New York, NY 10065, USA \\ ${ }^{3}$ Malaysian Palm Oil Board, Kajang, Selangor, Malaysia
}

"Corresponding author: Sanchez JA, Department of Biology, Brandeis University, Waltham, MA, 02454, USA, Tel: 1-781-736-3111; Fax: 1-781-736-3107; E-mail: sanchez@brandeis.edu

Received date: September 18, 2014; Accepted date: December 16, 2014; Published date: December 20, 2014

Copyright: (c) 2014 Osborne AE, et al. This is an open-access article distributed under the terms of the Creative Commons Attribution License, which permits unrestricted use, distribution, and reproduction in any medium, provided the original author and source are credited.

\section{Abstract}

Chronic use of 3'-Azido-3'-deoxythymidine (AZT) to treat HIVIAIDS causes mitochondrial dysfunction and the accumulation of mitochondrial mutations. These toxicities have been attributed to increased oxidative damage, among other mechanisms. Palm fruit juice (PFJ), also known as oil palm phenolics (OPP), is a water soluble byproduct of oil extraction from the fruit of the oil palm (Elaeis guineensis) that is rich in antioxidants and other phytochemicals. The capacity of PFJ to mitigate AZT mitochondrial genotoxicity (mutagenesis) as well as dosedependent cytotoxicity were measured in cultured HepG2 cells. In the presence of PFJ, AZT-induced mutations were $35 \%$ the number of mutations observed in samples treated with AZT alone in the three regions of the mitochondrial genome examined (HV2, CO2, and ND1). Co-treatment with PFJ increased cell survival in the presence of increasing doses of AZT by up to $350 \%$. These effects were not due to degradation or inactivation of AZT by PFJ. The discovery of the mitigating effects of PFJ provides a potential means of ameliorating AZT-induced mutations and possibly other long-term negative side effects of long-term AZT use.
\end{abstract}

Keywords: Mitochondria; LATE-PCR; Light-on/Lights-off probes; AZT toxicity; Palm fruit juice; Oil palm phenolics; Mutational load

\section{Introduction}

The nucleotide reverse transcriptase inhibitor (NRTI) $3^{\prime}$ Azido-3'-deoxythymidine (AZT, zidovudine) is widely used in the developing world to treat HIV/AIDS. AZT treatment, however, results in toxic side effects, including skeletal and cardiac myopathies, hyperlactatemia, and peripheral neuropathy [1-4]. Chronic AZT therapy is also associated with an increased incidence of mitochondria-related diseases such as type 2 diabetes and neurological disorders [5-7]. These side effects are secondary to the anti-retroviral activity of the drug and are consistent with AZT damage leading to mitochondrial dysfunction [8-10]. Because of its toxicity, AZT has been replaced by other less harmful NRTIs in most developed countries. Nonetheless, AZT remains an integral component of highly active anti-retroviral therapy (HAART) in the rest of the world. Mitochondrial damage and dysfunction resulting from AZT treatment remain of great concern in the treatment of HIV/AIDS.

The exact mechanism of AZT toxicity is not known. AZT toxicity could be due to mitochondrial dysfunction resulting from increased oxidative stress and/or mitochondria DNA mutations. Many clinical and experimental studies have shown mitochondrial dysfunction and increased oxidative stress following AZT treatment both in vitro and in vivo [11-13]. Consistent with these observations, AZT toxicity in cell culture, mice, and humans has been mitigated by co-treatment with individual antioxidants (vitamin $\mathrm{C}$, vitamin $\mathrm{E}$, resveratrol, etc.) [14-17]. There is now direct evidence that AZT causes mitochondrial DNA mutations [18-20].
This report describes the use of an antioxidant, palm fruit juice (PFJ), to mitigate AZT-induced mitochondria mutations and dosedependent cytotoxicity in HepG2 cells, the experimental system in which mitochondrial mutagenesis by AZT was recently reported [18]. Palm fruit juice is a water soluble by-product of oil extraction from the fruit of the oil palm (Elaeis guineensis) that is rich in antioxidant phenolics $[20,21]$. Palm fruit juice has high scavenging activity against hydrogen peroxide, the main contributor to reactive oxygen species (ROS) produced in mitochondria [20,22,23]. Palm fruit juice also exhibits various protective biological effects, including antiatherogenic, anti-inflammatory, anti-diabetic, and anti-neoplastic properties [24-31]. For example, dietary supplementation with PFJ deters the progression of type 2 diabetes, a disease associated with both mitochondrial dysfunction and chronic AZT treatment [29,31]. The results of the present study reveal that PFJ mitigated both AZTmutagenesis and dose-dependent cytotoxicity.

\section{Materials and Methods}

Detection of Mutations after AZT and PFJ Treatment for 30 Days Culture Conditions and DNA Preparation

Cell culture conditions and preparation of the mitochondrial DNA were the same as previously reported [18] except that HepG2 cells were also cultured in the presence or absence of $25 \mu \mathrm{g}$ gallic acid equivalents (GAE)/ml PFJ (a gift from the Malaysian Palm Oil Board). Twenty-five micrograms GAE per millilitre PFJ was the highest concentration that permits normal cell growth in conventional Eagle's Minimum Essential Medium (EMEM, ATCC Manassas, VA) used in the original experiments where AZT was shown to be mutagenic to mitochondria [18]. Buffered culture medium employed in the cytotoxicity study (see Supplemental Data) was not used for these 
Page 2 of 5

experiments to preserve the original culture conditions used to identify AZT-induced mutations [18]. The thirty-day incubation time for the genotoxicity study described here and in the original study [18] was based on reports that mitochondrial dysfunction in HepG2 cells caused by the physiologically relevant concentrations of AZT used in our study $(7 \mathrm{uM})$ requires long-term exposure (4 weeks) to develop [11].

Mitochondrial DNA was prepared as previously reported [32]. Briefly, one microliter of cultured cells (roughly 1000 cells) was lysed in $14 \mu \mathrm{l}$ of Quantilyse [33]. Samples were stored at $-20^{\circ} \mathrm{C}$.

\section{Cytotoxicity Tests}

HepG2 cultures were incubated with increasing concentrations of AZT $(0 \mu \mathrm{M}, 10 \mu \mathrm{M}, 30 \mu \mathrm{M}, 50 \mu \mathrm{M}, 70 \mu \mathrm{M}, 100 \mu \mathrm{M})$ in the presence or absence of $75 \mu \mathrm{g}$ GAE/ml PFJ in buffered media (see Supplemental Information). Seventy-five micrograms GAE per millilitre PFJ was used for these studies because it was the highest concentration of PFJ that did not arrest the growth of HepG2 cells after 25 day culture under buffered culture conditions (Supplemental Figure 1). Growth arrest observed at concentrations above $75 \mu \mathrm{g}$ GAE/ml PFJ is characteristic of the known cytostatic activity of PFJ against neoplastic cells such as HepG2 cells [34].

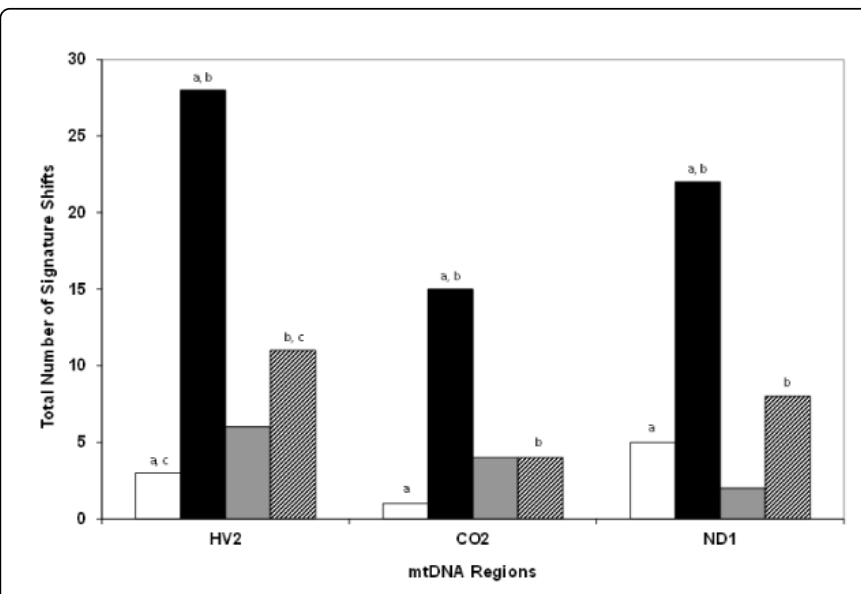

Figure 1: Palm Fruit Juice ameliorated AZT-induced mtDNA mutations. Thirty days of AZT treatment $(7 \mu \mathrm{M})$ also resulted in an increase in mutations in the mitochondrial genome, while cotreatment with $25 \mu \mathrm{g}$ GAE/ml PFJ reduced the number of mutations. Treatment with PFJ alone did not cause an increase in mutations. White $=$ Non-treated control, Black=AZT treated, Grey $=\mathrm{PFJ}$ treated, and Striped $=\mathrm{AZT}$ and PFJ treatment. ${ }^{*}$ represents those samples that had a p-value of 0.05 or less.

Cells were grown in 6-well plates at $37^{\circ} \mathrm{C}$ and $5 \% \mathrm{CO}_{2}$ in EMEM supplemented with $25 \mathrm{mM}$ HEPES, $10 \%$ fetal bovine serum (BioWest), 50 units $/ \mathrm{mL}$ Penicillin G, 50 units $/ \mathrm{mL}$ Streptomycin, and $0.25 \mu \mathrm{g} / \mathrm{mL}$ Amphotericin B (HyClone Antibiotic/Antimycotic Solution 100X). Media and additives were replenished every other day. Palm fruit juice was sterilized through a $0.2 \mu \mathrm{m}$ filter before addition to the media. Cell cultures were initially seeded with $1 \times 10^{5}$ cells/mL. Every 3-4 days cells were trypsinized and stained for membrane integrity using Trypan Blue (Sigma Aldrich, St. Louis, MO). The cells were then counted to measure cell growth and re-plated in fresh media at $1 \times 10^{5}$ cells $/ \mathrm{mL}$. Experiments were repeated three times.

\section{LATE-PCR Lights-On/Lights-Off Probe Assay for Mutation Detection}

\section{Reagents, primers, probes, and reaction conditions}

Lysed DNA samples were first diluted to the digital or near-digital level (1-5 copies) as described in Osborne et al. [32]. To avoid contamination, all PCR preparation was conducted in an ampliconfree hood in a clean room, all targets were kept separate from PCR reagents at all times, and PCR machines were kept in a separate room from the laboratory space as far away from the clean room as possible. PCR amplicons and products were never handled in the same laboratory space.

Primers, probes and amplification conditions used for the triplex LATE-PCR assay for mutation analysis of the mitochondrial hyper variable 2 (HV2) region of the D-Loop, the cytochrome c oxidase subunit 2 (CO2) gene, the $\mathrm{NADH}$ dehydrogenase, subunit 1 (ND1) gene were as previously described [18]. A single master mix was used for all experiments to ensure the reproducibility of the fluorescent signatures.

\section{Mutation analysis}

Mutations were scored as described by Osborne et al.. Briefly, sets of Lights-On/Lights-Off probes spanning the length of each amplicon were used to generate fluorescent signatures characteristic of each amplicon sequence. Changes in a fluorescent signature, i.e., shifts to lower or higher temperature or appearance or disappearance of peaks and valleys, relative to a reference signature were indicative of mutations. To obtain reference signatures for normal sequences, bulk reactions containing over 1000-copies of mtDNA each were amplified and the resulting fluorescent signatures were averaged together (for a more detailed discussion of fluorescent signature analysis see Osborne et al.). Shifted signatures that had more than two temperature points outside the confidence intervals of the reference signature were considered a true shift due to mutation rather than noise. These objective criteria for scoring mutations eliminated subjective bias during mutation assessment [18].

\section{Measurement of mtDNA copy number}

Measurement of mtDNA copy number was carried out as previously reported in Osborne et al. [18].

\section{Statistical analysis}

Statistical analysis of the cytotoxicity and genotoxicity data was carried out using Pearson's chi-square test. The Pearson's chi-square test establishes whether there is a significant difference between the expected and observed frequencies in one or more of the treatment categories. The test was performed using 95\% significance ( $p$-value of less 0.05).

\section{Results}

\section{Palm fruit juice mitigated AZT-induced mtDNA mutations}

In order to investigate whether increased oxidative stress caused by AZT leads to mtDNA mutations, AZT-induced mutations were measured in HepG2 cells treated for 30 days with $7 \mu \mathrm{M}$ AZT in the presence and absence of $25 \mu \mathrm{g}$ GAE/mL PFJ. Seven micromolar AZT is the peak plasma concentration in AZT-treated individuals previously found to induce mutations in HepG2 cells [18]. Three regions from the mitochondrial genome, $\mathrm{HV} 2, \mathrm{CO} 2$, and $\mathrm{ND} 1$, were examined for 
Page 3 of 5

mutations using a multiplex LATE-PCR Lights-On/Lights-Off assay at the digital or near digital levels [18]. A total of 352 wells with 1-5 genomes of mtDNA (an estimated 1,056 amplicons total) were evaluated.

AZT treatment alone caused up to a 9-fold increase in the number of random mutations in all three target genes compared to non-treated samples (p-values of $8 \times 10^{-6}, 0.0003$, and 0.0008 for $\mathrm{HV} 2, \mathrm{CO} 2$, and ND1 respectively; Figure 1). Palm fruit juice treatment alone was not mutagenic (Figure 1). Co-treatment with $7 \mu \mathrm{M}$ AZT and $25 \mu \mathrm{g}$ $\mathrm{GAE} / \mathrm{mL} \mathrm{PFJ}$ resulted in $35 \%$ the number of random mutations caused by AZT alone ( $p$-values of $0.005,0.008$, and 0.007 for HV2, CO2, and ND1 respectively; Figure 1). Measurements of the ratio of nuclear to mitochondrial genomes in PFJ and AZT co-treated samples showed that PFJ did not reduce the number of mitochondrial mutations by simply increasing the number of mitochondrial genomes (data not shown). These results demonstrate that PFJ ameliorated the mutagenic activity of AZT.

\section{Palm Fruit Juice mitigated dose-dependent AZT cytotoxicity in HepG 2 cells}

To investigate whether PFJ also mitigates AZT dose-dependent cytotoxicity, HepG2 cultures were incubated with various concentrations of AZT $(0 \mu \mathrm{M}, 10 \mu \mathrm{M}, 30 \mu \mathrm{M}, 50 \mu \mathrm{M}, 70 \mu \mathrm{M}, 100 \mu \mathrm{M})$ in the presence or absence of $75 \mu \mathrm{g} \mathrm{GAE} / \mathrm{mL}$ of PFJ. Increasing concentrations of AZT from $0 \mu \mathrm{M}$ to $100 \mu \mathrm{M}$ resulted in a progressive increase in cell death after 6 days in culture (Figure 2). Treatment with $100 \mu \mathrm{M}$ AZT declined cell viability by $90 \%$. Addition of $75 \mu \mathrm{g}$ $\mathrm{GAE} / \mathrm{mL} \mathrm{PFJ}$ resulted in increased protection against AZT-induced cell death from a minimal $12 \%$ improvement at $30 \mu \mathrm{M}$ AZT to about $350 \%$ improvement at $100 \mu \mathrm{M} \mathrm{AZT} \mathrm{(p-values} \mathrm{of} 0.04$ and 0.01 ) (Figure 2 ). These results demonstrate that inclusion of PFJ in the culture medium mitigated AZT's dose-dependent cytotoxicity.

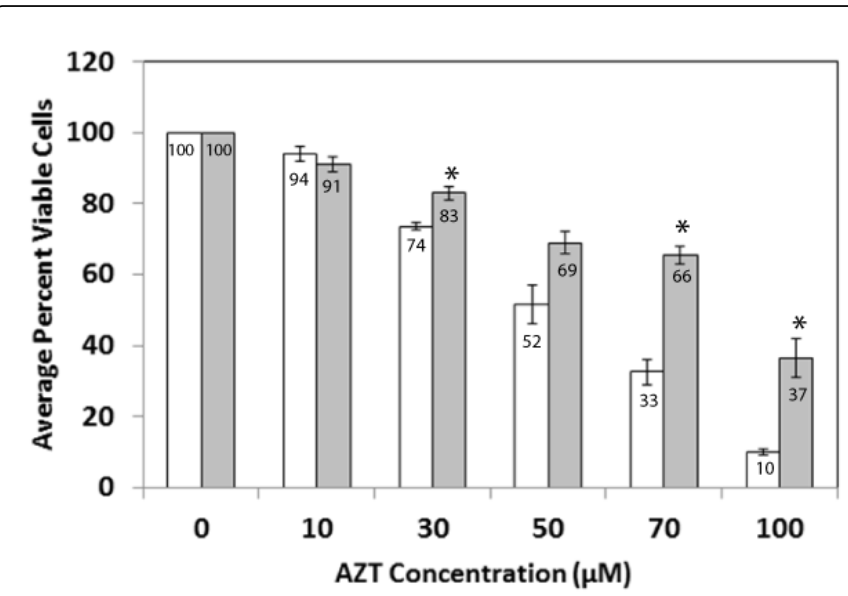

Figure 2: Co-treatment with PFJ reduced AZT dose dependent cytotoxicity after 6 days in HepG2 cells. Cell viability is plotted against co-treatment of increasing levels of AZT $(0 \mu \mathrm{M}, 10 \mu \mathrm{M}, 30$ $\mu \mathrm{M}, 50 \mu \mathrm{M}, 70 \mu \mathrm{M}$, and $100 \mu \mathrm{M})$ in the presence of $75 \mu \mathrm{g} \mathrm{GAE} / \mathrm{ml}$ $\mathrm{PFJ}$. White $=\mathrm{AZT}$ treated control and Grey $=\mathrm{AZT}$ and PFJ treated. ${ }^{\star}$ represents those samples that had a p-value of 0.05 or less. The error bars show the standard error.

\section{Palm Fruit Juice did not degrade AZT}

A possible explanation of the protective effects of PFJ on mutagenesis and cytotoxicity is that PFJ simply degraded AZT. This was not the case because day ten cultures showed that AZT modulated the cytostatic activity of PFJ. In the absence of AZT $75 \mu \mathrm{g}$ GAE/mL PFJ did not arrest HepG2 cell growth after 25 days in culture (Supplemental Figure 1). In contrast, co-treatment with AZT and PFJ arrested cell growth after ten days in culture, although cells remained viable (Table 1). These results showed that PFJ did not inactivate AZT and that the protective effect of PFJ were due to the activity of PFJ within HepG2 cells.

\begin{tabular}{|l|l|l|l|}
\hline $\begin{array}{l}\text { AZT }(\boldsymbol{\mu M}) \\
\text { No PFJ }\end{array}$ & $\begin{array}{l}\text { Day 10 Cell Conc. } \\
(\text { cells/ml) }\end{array}$ & $\begin{array}{l}\text { AZT }(\boldsymbol{\mu M})+ \\
\mathbf{7 5} \mu \mathrm{g} \text { GAE/mI PFJ }\end{array}$ & $\begin{array}{l}\text { Day 10 Cell Conc. } \\
(\text { cells } / \mathrm{ml})\end{array}$ \\
\hline 0 & $7.0 \times 105$ & 0 & $6.9 \times 105$ \\
\hline 10 & $5.6 \times 105$ & 10 & $5.5 \times 105$ \\
\hline 30 & $4.5 \times 105$ & 30 & $3.0 \times 105$ \\
\hline 50 & $2.4 \times 105$ & 50 & $1.8 \times 105$ \\
\hline 70 & $1.3 \times 105^{*}$ & 70 & $1.1 \times 105$ \\
\hline 100 & Dead & 100 & $1.1 \times 105$ \\
\hline
\end{tabular}

Table 1: High concentrations of AZT enhance the cytostatic effect of $75 \mu \mathrm{g}$ GAE/ml PFJ.

Day 6 culture cells were trypsinized and plated in fresh culture media with various concentrations of AZT in the absence or presence of $75 \mu \mathrm{g} \mathrm{GAE} / \mathrm{ml}$ PFJ at an initial concentration of $1 \times 10^{5}$ cells $/ \mathrm{ml}$. At day 10, cells were trypsinized again, stained with Trypan Blue, and counted four times per culture to measure the total concentration of cells. The results show that the same concentration of cells plated on Day 6 was recovered on Day 10 for $70 \mu \mathrm{M}$ AZT and $100 \mu \mathrm{M}$ AZT cotreated with $75 \mu \mathrm{g}$ GAE/ml PFJ and that cells remained viable. This phenomenon is characteristic of the cytostatic effect of $75 \mu \mathrm{g} \mathrm{GAE} / \mathrm{ml}$ PFJ on HepG2 cells observed after Day 20 culture in the absence of AZT (see Supplementary Figure 1) but occurred 15 days earlier. These data indicate that high concentrations of AZT enhanced the cytostatic effect of $75 \mu \mathrm{g}$ GAE/ml PFJ and that growth-arrested cells in the presence of PFJ continued to be protected from AZT cytotoxicity. In the presence of lower concentrations of AZT $(30 \mu \mathrm{M}$ and $50 \mu \mathrm{M}), 75$ $\mu \mathrm{g}$ GAE/ml PFJ exhibited slower cell growth suggestive of a partial PFJ cytostatic effect. ${ }^{*}=$ cells displayed an aberrant morphology (Figure 1).

\section{Discussion}

The results presented here demonstrate that addition of PFJ mitigated both AZT-induced mitochondrial mutagenesis and dosedependent cytotoxicity. Since AZT toxicity is related to mitochondrial dysfunction [8-10], the discovery that PFJ mitigated the genotoxic and cytotoxic effects of AZT suggests that PFJ exerts a protective effect on mitochondria. These studies expand the range of protective effects associated with PFJ [24-30].

\section{Protective effects of PFJ against AZT mutagenesis}

AZT treatment increases oxidative stress in cells and causes mutagenesis and dose-dependent cytotoxicity, but the biochemical basis for these phenomena remains unclear. Since PFJ is rich in 
antioxidants and many antioxidants are known to modulate Reactive Oxygen Species (ROS) [23] it is logical to postulate that PFJ suppresses mutations caused by ROS. However, PFJ only reduced the number of AZT-induced mutations by $65 \%$, suggesting that only a fraction of the mutations arise because of an increase in ROS and that the AZT also acts by ROS independent mechanisms. In fact, two hypotheses have been proposed to explain AZT-induced mutatagenesis. Mutations might arise from damage of mitochondrial DNA by ROS generated by AZT [35-38]. Alternatively, mutations may be due either to deleterious interaction between AZT and the mitochondrial DNA polymerase or to changes in nucleotide pools, either of which could alter the fidelity of mtDNA replication $[39,40]$. It is also possible that the highest permissible concentration of PFJ in non-buffered media has a limited antagonistic capacity.

Regardless of which mechanism is operative, AZT-induced DNA damage likely results in damage to mitochondrial proteins and this, in turn, probably further increases ROS production and accelerates the processes of mitochondrial DNA damage and dysfunction. Thus, independently of mechanism, the functional benefit of PFJ can be viewed as delaying the accumulation of mitochondrial DNA damage and the rate at which mitochondrial dysfunction is manifest.

\section{Protective effects of PFJ against AZT cytotoxicity}

The protective effects of antioxidants such as PFJ against AZT cytotoxicity has not been widely studied $[15,41]$. Palm fruit juice may attenuate AZT cytotoxicity by counteracting increased oxidative stress. Given the complex composition of PFJ $[42,43]$ a simple alternative hypothesis would be that some component(s) of PFJ might inactivate AZT in the culture media. The fact that AZT enhanced the cytostatic activity of PFJ argues against PFJ degrading AZT. Independent studies also show that AZT remains active in the presence of PFJ [23]. The cytostatic activity of PFJ appears to arise from changes in gene expression that lead to G1/S phase arrest [44]. Future studies will address how AZT interacts with cells to enhance the cytostatic effects of PFJ and how cell cycle arrest prevents AZT-induced cell death.

\section{Significance}

In contrast to other antioxidants used to mitigate AZT toxicity (such as vitamin E and resveratrol $[14,17]$ ) which are defined chemical species, PFJ is a complex mixture of chemicals. The experimental system described here could be used to identify the component(s) of $\mathrm{PFJ}$ responsible for the range of protective effects observed. The biomarkers used to evaluate the protective effects of PFJ (cytotoxicity and mitochondrial genotoxicity) could be used to assess other drugs and naturally derived phenolics for their effects on mitochondria.

The earlier findings that AZT treatment causes mutagenesis of mtDNA suggests that patients chronically treated with this drug as part of HAART might be experiencing continuous damage to their mitochondrial genomes $[18,20,45]$. Accumulation of mtDNA damage over time may explain the increased incidence of mitochondrialrelated diseases observed in these patients. The discovery that PFJ mitigates AZT toxicity suggests a possible approach to ameliorate or significantly delay the toxic side effects of AZT affecting millions of people.

\section{Acknowledgements}

This work was funded in part by the Malaysian Palm Oil Board to K.C.H and L.J.W. The Malaysian Palm Oil Board had no role in study design; in the collection, analysis, and interpretation of data; in the writing of the report; and in the decision to submit the paper for publication.

\section{References}

1. Aboud M, Elgalib A, Kulasegaram R, Peters B (2007) Insulin resistance and HIV infection: a review. Int J Clin Pract 61: 463-472.

2. Shikuma CM, Day LJ, Gerschenson M (2005) Insulin resistance in the HIV-infected population: the potential role of mitochondrial dysfunction. Curr Drug Targets Infect Disord 5: 255-262.

3. Samaras K, Wand H, Law M, Emery S, Cooper D, et al. (2007) Prevalence of metabolic syndrome in HIV-infected patients receiving highly active antiretroviral therapy using International Diabetes Foundation and Adult Treatment Panel III criteria: associations with insulin resistance, disturbed body fat compartmentalization, elevated C-reactive protein, and [corrected] hypoadiponectinemia. Diabetes Care 30: 113-119.

4. Barret B, Tardieu M, Rustin P, Lacroix C, Chabrol B, et al. (2003) Persistent mitochondrial dysfunction in HIV-1-exposed but uninfected infants: clinical screening in a large prospective cohort. AIDS 17: 1769-1785.

5. De Wit S, Sabin CA, Weber R, Worm SW, Reiss P, et al. (2008) Incidence and risk factors for new-onset diabetes in HIV-infected patients: the Data Collection on Adverse Events of Anti-HIV Drugs (D:A:D) study. Diabetes Care 31: 1224-1229.

6. Blümer RM, van Vonderen MG, Sutinen J, Hassink E, Ackermans M, et al. (2008) Zidovudine/lamivudine contributes to insulin resistance within 3 months of starting combination antiretroviral therapy. AIDS 22: 227-236.

7. Kakuda TN (2000) Pharmacology of nucleoside and nucleotide reverse transcriptase inhibitor-induced mitochondrial toxicity. Clin Ther 22: 685-708.

8. Lewis W, Gonzalez B, Chomyn A, Papoian T (1992) Zidovudine induces molecular, biochemical, and ultrastructural changes in rat skeletal muscle mitochondria. J Clin Invest 89: 1354-1360.

9. de Martino M, Zammarchi E, Filippi L, Donati MA, Mannelli F, et al. (1995) Redox potential status in children with perinatal HIV-1 infection treated with zidovudine. AIDS 9: 1381-1383.

10. Dykens JA, Will Y (2007) The significance of mitochondrial toxicity testing in drug development. Drug Discov Today 12: 777-785.

11. Kline ER, Bassit L, Hernandez-Santiago BI, Detorio MA, Liang B, et al. (2009) Long-term exposure to AZT, but not d4T, increases endothelial cell oxidative stress and mitochondrial dysfunction. Cardiovasc Toxicol 9: 1-12.

12. Hayakawa M, Ogawa T, Sugiyama S, Tanaka M, Ozawa T (1991) Massive conversion of guanosine to 8-hydroxy-guanosine in mouse liver mitochondrial DNA by administration of azidothymidine. Biochem Biophys Res Commun 176: 87-93.

13. Valenti D, Atlante A, Barile M, Passarella S (2002) Inhibition of phosphate transport in rat heart mitochondria by 3'-azido-3'deoxythymidine due to stimulation of superoxide anion mitochondrial production. Biochem Pharmacol 64: 201-206.

14. de la Asunción JG, del Olmo ML, Sastre J, Millán A, Pellín A, et al. (1998) AZT treatment induces molecular and ultrastructural oxidative damage to muscle mitochondria. Prevention by antioxidant vitamins. J Clin Invest 102: 4-9.

15. Gao RY, Mukhopadhyay $\mathrm{P}$, Mohanraj R, Wang $\mathrm{H}$, Horváth $\mathrm{B}$, et al. (2011) Resveratrol attenuates azidothymidine-induced cardiotoxicity by decreasing mitochondrial reactive oxygen species generation in human cardiomyocytes. Mol Med Rep 4: 151-155.

16. de la Asunción JG, Del Olmo ML, Gómez-Cambronero LG, Sastre J, Pallardó FV, et al. (2004) AZT induces oxidative damage to cardiac mitochondria: protective effect of vitamins $C$ and E. Life Sci 76: 47-56.

17. Zuena AR, Giuli C, Venerosi Pesciolini A, Tramutola A, Ajmone-Cat MA, et al. (2013) Transplacental exposure to AZT induces adverse 
neurochemical and behavioral effects in a mouse model: protection by $\mathrm{L}-$ acetylcarnitine. PLoS One 8: e55753.

18. Osborne AE, Rice JE, Sanchez JA, Wangh LJ (2013) AZT Treatment Increases mtDNA Mutations in HepG2 and CCD-1112Sk Cells J AIDS Clin Res 4: 250

19. Martin AM, Hammond E, Nolan D, Pace C, Den Boer M, et al. (2003) Accumulation of Mitochondrial DNA Mutations in Human Immunodeficiency Virus-Infected Patients Treated with NucleosideAnalogue Reverse-Transcriptase Inhibitors. Am J Hum Genet 72: 549-560.

20. Payne BA, Wilson IJ, Hateley CA, Horvath R, Santibanez-Koref M, et al. (2011) Mitochondrial aging is accelerated by anti-retroviral therapy through the clonal expansion of mtDNA mutations. Nat Genet 43 806-810.

21. Onyango I, Khan S, Miller B, Swerdlow R, Trimmer P, et al. (2006) Mitochondrial genomic contribution to mitochondrial dysfunction in Alzheimer's disease. J Alzheimers Dis 9: 183-193.

22. Cadenas E. (2004) Mitochondrial free radical production and cell signalling. Mol. Aspects. Med 23: 17-26.

23. Osborne AE, Sanchez JA, Wangh LJ, Sambanthamurthi R, Hayes KC (2014) Use of Palm Fruit Juice Shows that Oxidative Damage is not a Major Contributor to AZT-Induced Mitochondrial Mutations.

24. Naik P, Rozman HD, Bhat R (2013) Genoprotective effects of lignin isolated from oil palm black liquor waste. Environ Toxicol Pharmacol 36 135-141.

25. Leow SS, Sekaran SD, Tan Y, Sundram K, Sambanthamurthi R (2013) Oil palm phenolics confer neuroprotective effects involving cognitive and motor functions in mice. Nutr Neurosci 16: 207-217.

26. Leow SS, Sekaran SD, Sundram K, Tan Y, Sambanthamurthi R (2013) Oil palm phenolics attenuate changes caused by an atherogenic diet in mice. Eur J Nutr 52: 443-456.

27. Sambanthamurthi R, Tan Y, Sundram K, Abeywardena M, Sambandan TG, et al. (2011) Oil palm vegetation liquor: a new source of phenolic bioactives. Br J Nutr 106: 1655-1663.

28. Leow SS, Sekaran SD, Sundram K, Tan Y, Sambanthamurthi R (2011) Differential transcriptomic profiles effected by oil palm phenolics indicate novel health outcomes. BMC Genomics 12: 432.

29. Sambanthamurthi R, Tan Y, Sundram K, Hayes KC, Abeywardena M, et al. (2011) Positive outcomes of oil palm phenolics on degenerative diseases in animal models. Br J Nutr 106: 1664-1675.

30. Bolsinger J, Pronczuk A, Hayes KC (2013) Dietary carbohydrate dictates development of Type 2 diabetes in the Nile rat 24: 1945-1952.

31. Bolsinger J, Pronczuk A, Sambanthamurthi R, Hayes KC (2014) Antidiabetic effects of palm fruit juice in the Nile rat (Arvicanthis niloticus). Journal of Nutritional Science 3 .
32. Osborne A, Reis AH, Bach L, Wangh LJ (2009) Single-molecule LATEPCR analysis of human mitochondrial genomic sequence variations.

33. Pierce KE, Rice JE, Sanchez JA, Wangh LJ (2002) QuantiLyse: reliable DNA amplification from single cells. BioTechniques 32: 1106-1111.

34. Shamala Devi Sekaran, Soon-Sen Leow, Najwa Abobaker, Kok Keng Tee1, Kalyana Sundram, et al. (2010) Effects of oil palm phenolics on tumor cells in vitro and in vivo. African Journal of Food Science 4: 495-502.

35. Barile M, Valenti D, Hobbs GA, Abruzzese MF, Keilbaugh SA, et al. (1994) Mechanisms of toxicity of 3'-azido-3'-deoxythymidine. Its interaction with adenylate kinase. Biochem Pharmacol 48: 1405-1412.

36. Valenti D, Barile M, Passarella S (2000) AZT inhibition of the ADP/ATP antiport in isolated rat heart mitochondria. Int J Mol Med 6: 93-96.

37. Valenti D, Atlante A, Barile M, Passarella S (2002) Inhibition of phosphate transport in rat heart mitochondria by 3'-azido-3'deoxythymidine due to stimulation of superoxide anion mitochondrial production. Biochem Pharmacol 64: 201-206.

38. Modica-Napolitano JS (1993) AZT causes tissue-specific inhibition of mitochondrial bioenergetic function. Biochem Biophys Res Commun 194: 170-177.

39. Bridges EG, Faraj A, Sommadossi JP (1993) Inhibition of mammalian DNA polymerase-associated $3^{\prime}$ to $5^{\prime}$ exonuclease activity by $5^{\prime}$ monophosphates of 3'-azido-3'-deoxythymidine and 3'-amino-3'deoxythymidine. Biochem Pharmacol 45: 1571-1576.

40. Vela JE, Miller MD, Rhodes GR, Ray AS (2008) Effect of nucleoside and nucleotide reverse transcriptase inhibitors of HIV on endogenous nucleotide pools. Antivir Ther 13: 789-797.

41. García-de-la-Asunción J, Gómez-Cambronero LG, Del Olmo ML, Pallardó FV, Sastre J, et al. (2007) Vitamins C and E prevent AZTinduced leukopenia and loss of cellularity in bone marrow. Studies in mice. Free Radic Res 41: 330-334.

42. Balasundram N, Ai TY, Sambanthamurthi R, Sundram K, Samman S (2005) Antioxidant properties of palm fruit extracts. Asia Pac J Clin Nutr 4: 319-324.

43. Balasundram N, Bubb W, Sundram K, Samman S (2003) Antioxidants from palm (Elaeis guineensis) fruit extracts. Asia Pac J Clin Nutr 12: S37.

44. Sambanthamurthi R, Tan Y, Sundram K, Hayes KC, Abeywardena M, et al. (2011) Positive outcomes of oil palm phenolics on degenerative diseases in animal models. Br J Nutr 106: 1664-1675.

45. Martin AM, Hammond E, Nolan D, Pace C, Den Boer M, et al. (2003) Accumulation of Mitochondrial DNA Mutations in Human Immunodeficiency Virus-Infected Patients Treated with NucleosideAnalogue Reverse-Transcriptase Inhibitors. Am J Hum Genet 72: $549-560$. 